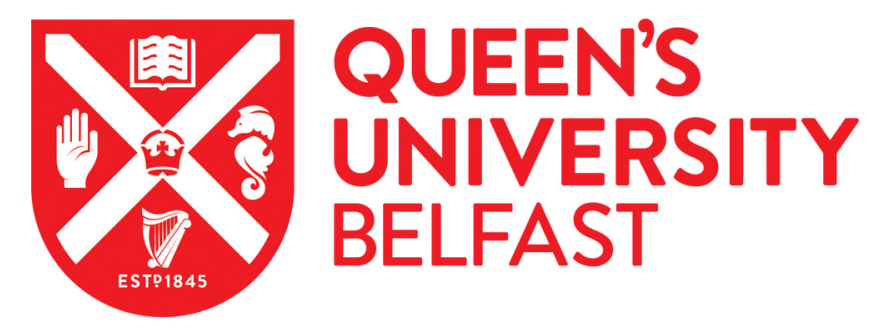

\title{
Landauer's Principle in Multipartite Open Quantum System Dynamics
}

Lorenzo, S., McCloskey, R., Ciccarello, F., Paternostro, M., \& Palma, G. M. (2015). Landauer's Principle in Multipartite Open Quantum System Dynamics. Physical Review Letters, 115(12), [120403].

https://doi.org/10.1103/PhysRevLett.115.120403

\section{Published in:}

Physical Review Letters

Document Version:

Publisher's PDF, also known as Version of record

Queen's University Belfast - Research Portal:

Link to publication record in Queen's University Belfast Research Portal

Publisher rights

(C) 2015 American Physical Society

\section{General rights}

Copyright for the publications made accessible via the Queen's University Belfast Research Portal is retained by the author(s) and / or other copyright owners and it is a condition of accessing these publications that users recognise and abide by the legal requirements associated with these rights.

Take down policy

The Research Portal is Queen's institutional repository that provides access to Queen's research output. Every effort has been made to ensure that content in the Research Portal does not infringe any person's rights, or applicable UK laws. If you discover content in the Research Portal that you believe breaches copyright or violates any law, please contact openaccess@qub.ac.uk. 


\title{
Landauer's Principle in Multipartite Open Quantum System Dynamics
}

\author{
S. Lorenzo, ${ }^{1,2,3}$ R. McCloskey, ${ }^{4}$ F. Ciccarello,${ }^{5}$ M. Paternostro, ${ }^{4}$ and G. M. Palma ${ }^{5}$ \\ ${ }^{1}$ Dipartimento di Fisica e Chimica, Università degli Studi di Palermo, Via Archirafi 36, I-90123 Palermo, Italy \\ ${ }^{2}$ Dipartimento Fisica, Università della Calabria, 87036 Arcavacata di Rende (CS), Italy \\ ${ }^{3}$ INFN - Gruppo collegato di Cosenza, Cosenza, Italy \\ ${ }^{4}$ Centre for Theoretical Atomic, Molecular, and Optical Physics, School of Mathematics and Physics, \\ Queen's University, Belfast BT7 1NN, United Kingdom \\ ${ }^{5}$ NEST, Istituto Nanoscienze-CNR and Dipartimento di Fisica, Università degli Studi di Palermo, \\ via Archirafi 36, I-90123 Palermo, Italy
}

(Received 10 April 2015; revised manuscript received 3 July 2015; published 16 September 2015)

\begin{abstract}
We investigate the link between information and thermodynamics embodied by Landauer's principle in the open dynamics of a multipartite quantum system. Such irreversible dynamics is described in terms of a collisional model with a finite temperature reservoir. We demonstrate that Landauer's principle holds, for such a configuration, in a form that involves the flow of heat dissipated into the environment and the rate of change of the entropy of the system. Quite remarkably, such a principle for heat and entropy power can be explicitly linked to the rate of creation of correlations among the elements of the multipartite system and, in turn, the non-Markovian nature of their reduced evolution. Such features are illustrated in two exemplary cases.
\end{abstract}

Logical irreversibility and heat dissipation are linked through the relation provided by Landauer in 1961 [1]: The erasure of information on the state of a system is concomitant with the dissipation of heat into the surroundings. In turn, such heat is lower bounded by a change in the information-theoretic entropy of the system. Landauer's principle, which has been recently tested [2], was a building block of remarkable advances in our understanding of thermodynamics and its links with logical irreversibility and information theory [3].

Recent progress in the quantum approach to nonequilibrium statistical mechanics $[4,5]$ has made the tracking of quantities such as heat, work, and entropy possible in an experimentally viable way. In turn, this has enabled the test bed demonstration of the link between information and energy in quantum systems subjected to elementary quantum computation protocols [6]. The relation between information and the thermodynamic costs of quantum operations has been extensively addressed in the recent past. Notable examples include Refs. [7] (see [8] for a recent overview). Techniques of quantum statistical mechanics have been used to prove that a finite-size environment can provide tighter bounds to the heat generated in an erasure process $[9,10]$. The validity of Landauer's principle has been verified for a quantum harmonic oscillator strongly coupled to a bath of bosonic modes [11]. However, such conceptual and experimental progress has not yet resulted in a satisfactory microscopic quantum framework able to account for the emergence of Landauer's principle.

In this Letter we provide a derivation of a Landauer-like principle that addresses the heat and entropy fluxes implied in an erasure process described by a collision-based picture of open-system dynamics [12]. Such a microscopic formulation has been used to examine the process of thermalization of a quantum system in contact with a nonzero temperature bath and to investigate the link between non-Markovianity and quantum correlations [13-15]. Here, we bring together these two perspectives: We analyze the process of information-to-energy conversion in collisional models that make use of general, nonrestrictive assumptions and effective mechanisms to describe the heat dissipation into an environment. Our Letter thus aims at bridging the gap highlighted above and contributes to the ongoing research for a satisfactory foundation of Landauer's principle. The advantages of our approach are manifold. First, modeling the irreversible dynamics of a system by a collisional model where, at each step, the environment is reset in a thermal state enables the formulation of Landauer's principle in terms of (heat and entropy) powers. Second, we show that the amount of heat dissipated by a multipartite quantum system is lower bounded by the correlations established among the elements of the system itself. Finally, the flexibility of the picture adopted here allows us to unveil interesting links between the violation of Landauer's principle and the emergence of memory-bearing effects leading to non-Markovian dynamics.

Erasure through thermalization.-We focus on the thermalization process of a system $\mathcal{S}$ in contact with an environment $\mathcal{R}$ endowed with a large number of degrees of freedom, which can thus be treated as a bath [12]. In our model $\mathcal{R}$ consists of a collection of $N$ identical noninteracting elements $\mathcal{R}_{n}$, hereafter dubbed "subenvironments," each of which is assumed to be in a thermal state. The bath is, therefore, in the product thermal state $\eta=\bigotimes_{n=1}^{N} \eta_{n}^{\text {th }}$, 
with $\eta_{n}^{\text {th }}=e^{-\beta \hat{H}_{\mathcal{R}_{n}}} / \operatorname{Tr}\left[e^{-\beta \hat{H}_{\mathcal{R}_{n}}}\right], \hat{H}_{\mathcal{R}_{n}}$ the free Hamiltonian of the $n$th subenvironment, and $\beta$ the inverse temperature. We call $\hat{H}_{\mathcal{S}}$ the free Hamiltonian of the system and, with no loss of generality, we assume $\hat{H}_{\mathcal{R}_{n}}=\hat{H}_{\mathcal{R}}$ for any $n$. The system interacts with the environment via a sequence of pairwise collisions with individual subenvironments. The assumption of a big reservoir implies that the system never interacts twice with the same subenvironment; therefore, at each collision the state of the subenvironment is refreshed and the system always interacts with subenvironments in a thermal state. Each collision is described by a unitary operator $\hat{U}=e^{-i g \hat{V} \tau}$, where $g$ is a coupling constant, $\tau$ is the collision time, and $\hat{V}=\sum_{k} \hat{S}_{k} \otimes \hat{R}_{k}$ is the (dimensionless) $\mathcal{S}-\mathcal{R}_{n}$ interaction Hamiltonian $\left[\hat{S}_{k}\left(\hat{R}_{k}\right)\right.$ are Hermitian operators acting on $\mathcal{S}\left(\mathcal{R}_{n}\right)$ ]. The information initially encoded in $\rho$ is gradually diluted into $\mathcal{R}$. After the $(n+1)$ th collisions, we find the states $\rho_{n+1}$ of the system and $\eta_{n+1}^{\prime}$ of the environment,

$$
\begin{gathered}
\rho_{n+1}=\operatorname{Tr}_{\mathcal{R}}\left[\hat{U} \rho_{n} \otimes \eta_{n+1}^{\mathrm{th}} \hat{U}^{\dagger}\right]=\Phi\left[\rho_{n}\right], \\
\eta_{n+1}^{\prime}=\operatorname{Tr}_{\mathcal{S}}\left[\hat{U} \rho_{n} \otimes \eta_{n+1}^{\mathrm{th}} \hat{U}^{\dagger}\right]=\Lambda_{n}\left[\eta_{n+1}^{\mathrm{th}}\right],
\end{gathered}
$$

where $\Phi\left(\Lambda_{n}\right)$ is a completely positive trace-preserving (CPTP) map on $\mathcal{S}(\mathcal{R})$ [16]. Unlike $\Phi$, map $\Lambda_{n}$ does depend on $n$ through $\rho_{n}$. A formal definition of mean heat flux and work is obtained considering infinitesimal changes of internal energy as $\delta \operatorname{Tr}[\mu \hat{H}]=\operatorname{Tr}[\mu \delta \hat{H}]+\operatorname{Tr}[\delta \mu \hat{H}]$, where $\mu$ is the density matrix of a system with Hamiltonian $\hat{H}$ [17]. The first term is identified with the average work done on or by the system; the second, which disappears for a unitary evolution, is a heat flux. Such arguments have been more recently reprised in Refs. [18]. In this sense, based on Eqs. (1) and (2), the corresponding energy variation of the system $\Delta E_{n+1}$ and heat exchange with reservoir $\Delta Q_{n+1}$, are given by

$$
\begin{aligned}
\Delta E_{n+1} & =\operatorname{Tr}\left[\hat{H}_{\mathcal{S}}(\Phi-\llbracket)\left[\rho_{n}\right]\right], \\
\Delta Q_{n+1} & =\operatorname{Tr}\left[\hat{H}_{\mathcal{R}}\left(\Lambda_{n}-\llbracket\right)\left[\eta^{\mathrm{th}}\right]\right] .
\end{aligned}
$$

We next assume $\left\langle\hat{R}_{k}\right\rangle_{\eta_{k}^{\text {th }}}=\operatorname{Tr}_{\mathcal{R}}\left[\hat{R}_{k} \eta_{k}^{\text {th }}\right]=0$ (this is possible, without affecting our results, by moving into an interaction representation with respect to a suitably redefined local Hamiltonian of $\mathcal{S}$, done without affecting our results). Assuming that each collision lasts a short time, we can expand $(\Phi-\llbracket)$ in series up to second order in $y=g \tau$ to find $\Delta \rho_{n+1}=(\Phi-\llbracket) \rho_{n}=\mathcal{K}_{2} \rho_{n}$, with $\mathcal{K}_{2}=\operatorname{Tr}_{\mathcal{R}}\left[\hat{V}\left(\rho_{n} \otimes \eta^{\text {th }}\right) \hat{V}-\right.$ $\left.\left\{\hat{V}^{2}, \rho_{n} \otimes \eta^{\text {th }}\right\} / 2\right]$. This gives us

$$
\begin{aligned}
& \Delta E_{n+1}=g^{2} \tau^{2} \sum_{k j}\left\langle\hat{R}_{k} \hat{R}_{j}\right\rangle_{\eta^{\mathrm{th}}}\left\langle\hat{S}_{k} \hat{H}_{\mathcal{S}} \hat{S}_{j}-\frac{1}{2}\left\{\hat{S}_{k} \hat{S}_{j}, \hat{H}_{\mathcal{S}}\right\}\right\rangle_{\rho_{n}}, \\
& \Delta Q_{n+1}=g^{2} \tau^{2} \sum_{k j}\left\langle\hat{S}_{k} \hat{S}_{j}\right\rangle_{\rho_{n}}\left\langle\hat{R}_{k} \hat{H}_{\mathcal{R}} \hat{R}_{j}-\frac{1}{2}\left\{\hat{R}_{k} \hat{R}_{j}, \hat{H}_{R}\right\}\right\rangle_{\eta^{\text {th }}} .
\end{aligned}
$$

In the limit $\tau \simeq 0$ and $n \gg 1$, the quantity $t=n \tau$ becomes a continuous variable, $\rho_{n} \rightarrow \rho(t)$ and $\left(\rho_{n+1}-\rho_{n}\right) / \tau \rightarrow \dot{\rho}$, so that we get the master equation (ME) $\dot{\rho}=\mathcal{K}_{2}[\rho(t)]$. Likewise, Eqs. (3) and (4) become

$$
\begin{aligned}
& \dot{E}=\gamma \sum_{k, j}\left\langle\hat{R}_{k} \hat{R}_{j}\right\rangle_{\eta^{\mathrm{th}}}\left\langle\hat{S}_{k} \hat{H}_{\mathcal{S}} \hat{S}_{j}-\frac{1}{2}\left\{\hat{S}_{k} \hat{S}_{j}, \hat{H}_{S}\right\}\right\rangle_{\rho}, \\
& \dot{Q}=\gamma \sum_{k, j}\left\langle\hat{S}_{k} \hat{S}_{j}\right\rangle_{\rho}\left\langle\hat{R}_{k} \hat{H}_{\mathcal{R}} \hat{R}_{j}-\frac{1}{2}\left\{\hat{R}_{k} \hat{R}_{j}, \hat{H}_{R}\right\}\right\rangle_{\eta^{\mathrm{th}}},
\end{aligned}
$$

where we have defined the rate $\gamma=g^{2} \tau$, achieved by taking both $g \rightarrow \infty$ and $\tau \rightarrow 0$ so that $g^{2} \tau$ is a constant. By doing so, we would guarantee the system-environment effects to persist (different from what is obtained by taking $\tau \rightarrow 0$ ). As discussed in Refs. $[13,14]$, with the assumption on $\left\langle\hat{R}_{k}\right\rangle_{\eta}$ invoked above, such a continuous-time limit is fully consistent and legitimate. By construction, $\rho(t)$ evolves according to a CPTP dynamical map $[12,16]$. We now focus on energy-conserving $\mathcal{S}-\mathcal{R}$ interactions and assume $\left[\hat{U},\left(\hat{H}_{\mathcal{S}}+\hat{H}_{\mathcal{R}}\right)\right]=0$. Hence, $\dot{Q}=-\dot{E}$ and the stationary state of the system is the Gibbs state at the same initial temperature of the bath, i.e., $\rho^{\mathrm{eq}}=e^{-\beta H_{\mathcal{S}}} / \operatorname{Tr}\left[e^{-\beta H_{\mathcal{S}}}\right]$. In this case, the stationary state is such that $\dot{E}=\dot{Q}=0$, which is not verified by non-energy-conserving models. In such cases, the steady state corresponds to a constant flux of heat into or out of the system.

The relative entropy $S\left(\rho \mid \rho^{\mathrm{eq}}\right)$ between the state at time $t$ and the stationary state obeys the relation $\dot{S}\left(\rho \mid \rho^{\mathrm{eq}}\right)=$ $-\dot{S}(\rho)+\beta \dot{E}[19]$. Because of the nonincreasing nature of the relative entropy under CPTP maps [20], we obtain

$$
\beta \dot{Q}(t) \geq \dot{\tilde{S}}(\rho),
$$

where $\tilde{S}(\rho)=-S(\rho)$. This embodies an open-system formulation of Landauer's principle for the heat and entropy fluxes. We can thus lower bound the flow of heat dissipated into the environment with the rate of change of a key information-theoretical quantity. Remarkably, our formulation enables the assessment of the nature of the opensystem dynamics of $\mathcal{S}$. The power of our formalism is thus twofold: On one hand, it allows for a time-resolved analysis of the erasure-by-thermalization process. On the other hand, it elucidates the role of correlations in the efficiency of information-erasure processes. This is illustrated by two examples of irreversible collisional dynamics. We first analyze a cascaded system where the subenvironments interact in sequence with the elements of a bipartite system, chosen here for ease of presentation. In this scenario, the environment is "recycled" to erase the information in the two subsystems before being damped. We show that the efficiency of information erasure is limited by the buildup of intersystem correlations. In the second example, which we dub "indirect," we consider a system interacting with an ancilla whose state is then erased by the environment. In this case, we show the appearance of local violation of Landauer's principle due to the onset of memory effects.

Information erasure in cascaded systems.-For now, we consider the system $\mathcal{S}$ as bipartite and consisting of two 
(a)

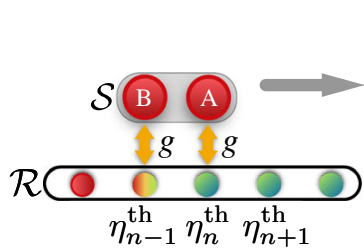

(b)

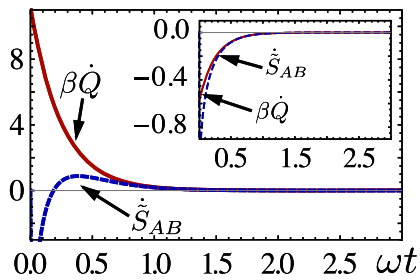

(c)

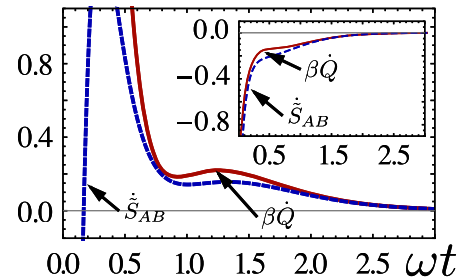

(d)

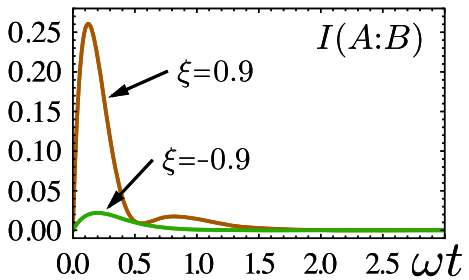

FIG. 1 (color online). (a) Sketch of the recycled environment setup: After colliding with $\mathcal{S}_{A}$, subenvironment $\mathcal{R}_{n}$ (prepared in $\eta_{n}^{\text {th }}$ ) is used to erase the state of $\mathcal{S}_{B}$. By tracing out the subenvironments, a Markovian ME is achieved for $\mathcal{S}$. (b) Heat flux and entropy for a system composed of two qubits erased by independent baths (no cascade) for $\xi=0.9$ and $\xi=-0.9$ (inset). (c) Heat and entropy flux for the cascade model. We have set $\gamma / \omega=1$ with $\mathcal{S}$ initially prepared in $|\uparrow \uparrow\rangle$ at $\xi=0.9$ and $\xi=-0.9$ (inset). (d) Mutual information $I(A: B)$ between the subsystems in the same case of panels (b) and (c) for $\xi=0.9$ (solid brown line) and $\xi=-0.9$ (dashed green line). Heat fluxes are expressed in unit of $\omega \gamma$.

identical subsystems $\mathcal{S}_{A, B}$. Each subenvironment $\mathcal{R}_{n}$ collides with $\mathcal{S}_{A}$ first and $\mathcal{S}_{B}$ later. Each collision is modeled as a unitary process with associated evolution operator $\hat{U}_{X}=e^{-i g \hat{V}_{X} \tau},(X=A, B)$ and generator $\hat{V}_{X}=\sum_{k} \hat{S}_{X k} \otimes$ $\hat{R}_{k}$ (here $\hat{S}_{X k}$ acts on subsystem $X$ ). A "step" consists of an $\mathcal{S}_{A}-\mathcal{R}_{n}$ collision followed by an $\mathcal{S}_{B}-\mathcal{R}_{n}$ one. Hence, the joint $\mathcal{S}-\mathcal{R}$ state at step $n+1$ is given by $\sigma_{n+1}=$ $\hat{U}_{B} \hat{U}_{A} \rho_{n} \eta^{\text {th }} \hat{U}_{A}^{\dagger} \hat{U}_{B}^{\dagger} \quad\left(\rho_{n} \quad\right.$ is the joint $\mathcal{S}_{A}-\mathcal{S}_{B} \quad$ state $)$. Equations (1) and (2) thus still hold with $\hat{U}=\hat{U}_{B} \hat{U}_{A}$.

Through a procedure analogous to the one outlined earlier, it can be shown that in the continuous-time limit the bipartite system $\mathcal{S}$ is governed by the ME [13]

$$
\dot{\rho}=\sum_{X=A, B} \mathcal{L}_{X}[\rho]+\mathcal{D}_{A B}[\rho],
$$

where superoperators $\mathcal{L}_{X}$ and $\mathcal{D}_{A B}$ are defined by

$$
\begin{aligned}
& \mathcal{L}_{X}[\rho]=\gamma \sum_{k j}\left\langle\hat{R}_{k} \hat{R}_{j}\right\rangle_{\eta^{\mathrm{th}}}\left(\hat{S}_{X j} \rho \hat{S}_{X k}-\frac{1}{2}\left\{\hat{S}_{X k} \hat{S}_{X j}, \rho\right\}\right), \\
& \mathcal{D}_{A B}[\rho]=\gamma \sum_{k j}\left\langle\hat{R}_{j} \hat{R}_{k}\right\rangle_{\eta^{\mathrm{th}}}\left[\hat{S}_{A k} \rho, \hat{S}_{B j}\right]+\left\langle\hat{R}_{k} \hat{R}_{j}\right\rangle_{\eta^{\mathrm{th}}}\left[\hat{S}_{B j}, \rho \hat{S}_{A k}\right] .
\end{aligned}
$$

Subsystems $\mathcal{S}_{A}$ and $\mathcal{S}_{B}$ jointly undergo a Markovian dynamics. This is true also for the dynamics of $\mathcal{S}_{A}$, given that it collides with "fresh" subenvironments every time [21]. However, in general, $\mathcal{S}_{B}$ is subjected to nonMarkovian evolution.

As before, given that no work is performed on the system, we identify the variation rate of the internal energy of the system $\dot{E}$ with $\Delta E_{n+1}=\operatorname{Tr}\left[\hat{H}_{\mathcal{S}}(\Phi-\llbracket)\left[\rho_{n}\right]\right]$. Using Eq. (8) and following an approach similar to the one presented above, it is possible to write explicitly the expression for the heat transferred at each step of the collision-based process, as shown in Ref. [22]. By focusing again on energy-conserving system-environment interactions, the identity $\dot{Q}=-\dot{E}$ holds and the system thermalizes at the same temperature of the environment. Accordingly, in light of the absence of any direct $\mathcal{S}_{A}-\mathcal{S}_{B}$ coupling, the system asymptotically reaches the stationary state $\rho^{\mathrm{eq}}=\rho_{A}^{\mathrm{eq}} \otimes \rho_{B}^{\mathrm{eq}}=e^{-\beta H_{\mathcal{S}_{A}}} \otimes e^{-\beta H_{\mathcal{S}_{B}}} /\left(Z_{A} Z_{B}\right)$ (here $Z_{X}=\operatorname{Tr}\left[e^{-\beta \hat{H}_{\mathcal{S}_{X}}}\right]$ ); i.e., the factorized state of locally thermal states at the same temperature. Indeed, despite the fact that $\mathcal{S}_{B}$ evolution depends on $\mathcal{S}_{A}$ (thus determining its non-Markovian character), $\mathcal{S}_{A}$ thermalizes with $\mathcal{R}$ in a fully Markovian fashion. Thereby, asymptotically, the $\mathcal{S}_{A}-\mathcal{R}_{n}$ collisions do not change the $\mathcal{R}_{n}$ state. Thus, in the same limit, $\mathcal{S}_{B}$ will also collide with subenvironments that are still in state $\eta^{\text {th }}$, forcing it to thermalize at the temperature of the environment. We remark that the bound stated in Eq. (7) clearly holds in the present situation, provided that $Q$ and $\tilde{S}(\rho)$ refer to system $\mathcal{S}$.

In order to show that recycling the environment affects the erasure efficiency, we use the definition of quantum mutual information $\mathcal{I}\left(\mathcal{S}_{A}: \mathcal{S}_{B}\right)=S\left(\rho_{\mathcal{S}_{A}}\right)+S\left(\rho_{\mathcal{S}_{B}}\right)-S(\rho)$ and conditional quantum entropy $S_{\mathcal{S}_{A} \mid \mathcal{S}_{B}}=S(\rho)-S\left(\rho_{\mathcal{S}_{A}}\right)$ [24] to get $-\dot{S}(\rho)=-2 \dot{S}\left(\rho_{\mathcal{S}_{A}}\right)+\dot{S}_{\mathcal{S}_{A} \mid \mathcal{S}_{B}}-\dot{S}_{\mathcal{S}_{B} \mid \mathcal{S}_{A}}+\dot{\mathcal{I}}\left(\mathcal{S}_{A}: \mathcal{S}_{B}\right)$ and, in turn,

$$
\beta \dot{Q} \geq 2 \dot{\tilde{S}}\left(\rho_{\mathcal{S}_{A}}\right)+\dot{S}_{\mathcal{S}_{A} \mid \mathcal{S}_{B}}-\dot{S}_{\mathcal{S}_{B} \mid \mathcal{S}_{A}}+\dot{I}\left(\mathcal{S}_{A}: \mathcal{S}_{B}\right)
$$

This shows that the erasure efficiency in cascaded systems depends on the rate at which correlations between $\mathcal{S}_{A}$ and $\mathcal{S}_{B}$ are established. Indeed, the last three terms in the right-hand side of Eq. (11) are null only for product states. Incidentally, the rate $2 \dot{\tilde{S}}\left(\rho_{\mathcal{S}_{A}}\right)$ is that of two identical states erased by independent reservoirs.

Our arguments are straightforwardly extended to the multipartite case. Using the correlation information $\mathcal{I}_{N}=$ $\sum_{i=1}^{N} S_{i}-S_{1, \ldots, N}$ [25], which extends mutual information to an $N$-partite system, Eq. (11) is extended to the erasure of $N$ copies as

$\beta \dot{Q}>N \dot{\tilde{S}}\left(\rho_{1}\right)+(N-1) \dot{S}_{1 \mid 2, \ldots, N}-\sum_{k=2}^{N} \dot{S}_{k \mid 1 \ldots}+\dot{\mathcal{I}}_{N}$.

We now illustrate the results described above with a specific example. We consider an all-qubit configuration where $\hat{H}_{\mathcal{S}}=(\omega / 2) \sum_{X=A, B} \hat{\sigma}_{X z}$ and $\hat{H}_{\mathcal{R}_{n}}=(\omega / 2) \hat{\sigma}_{\mathcal{R}_{n} z}$, with $\left\{\hat{\sigma}_{\ell i}\right\} \quad(i=x, y, z)$ the Pauli operators of qubit $\ell=X, \mathcal{R}_{n}$. Each subenvironment is prepared in the thermal state $\eta^{\text {th }}=[(1-\xi) / 2]|\uparrow\rangle\langle\uparrow|+[(1+\xi) / 2]| \downarrow\rangle\langle\downarrow|$, 
(a)

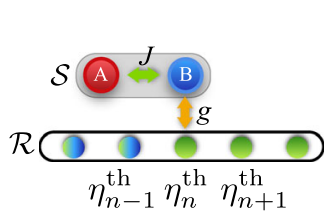

(b)

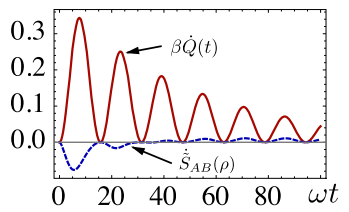

(c)

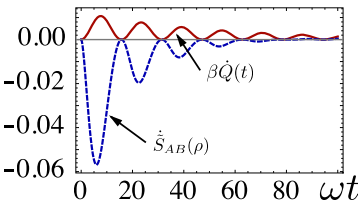

(d)

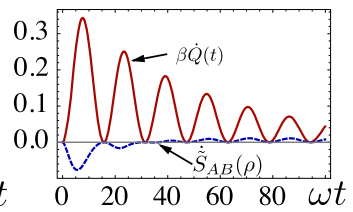

(e)

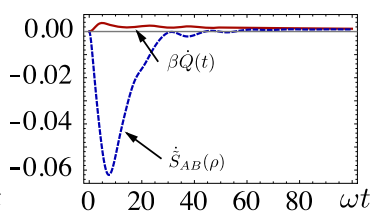

FIG. 2 (color online). (a) Sketch of the indirect-erasure protocol: The bipartite system $\mathcal{S}$ consists of subsystems $\mathcal{S}_{A}$ and $\mathcal{S}_{B}$. After $\mathcal{S}_{B}$ interacts with the $n$th subenvironment (prepared in $\eta_{n}^{\text {th }}$ ), it collides with $\mathcal{S}_{A}$ and is then directed to element $\mathcal{R}_{n+1}$. $\mathcal{S}_{B}$ thus bridges the erasure process undergone by $\mathcal{S}_{A}$, which experiences a non-Markovian evolution. (b)-(e): Total heat flow $\beta \dot{Q}$ against the entropy change rate $\tilde{S}$ for qubit-qubit [(b),(c)] and qubit-harmonic oscillator [(d),(e)] configurations. We have set $J / \omega=0.1$ and $\gamma_{g} / \omega=0.01$, taking $\mathcal{S}_{B}$ at the same temperature of the environment, $\beta=10[(\mathrm{~b}),(\mathrm{d})]$ and $\beta=0.5[(\mathrm{c}),(\mathrm{e})]$. A study against the value of $J$ and $\gamma_{g}$ is reported in Ref. [22].

where $\{|\uparrow\rangle,|\downarrow\rangle\}$ are the eigenstates of $\hat{\sigma}_{z}$ with $\xi=\tanh (\beta \omega / 2)$. The $\mathcal{S}_{X}-\mathcal{R}_{n}$ interaction Hamiltonian is of the isotropic $X X$ form, i.e., $\hat{V}_{X}=\sum_{i=x, y} \hat{\sigma}_{X i} \otimes \sigma_{\mathcal{R}_{n} i}$. In Ref. [22] we present the form taken by Eq. (8) in this case and show that the evolution of $\mathcal{S}_{A}$ is Markovian and not causally related to $\mathcal{S}_{B}$. Instead, the $\mathcal{S}_{B}$ dynamics depend crucially on the state of $\mathcal{S}_{A}$, thus being steered by previous subsystem-environment collisions. The behavior of $\beta \dot{Q}$ is compared to $\dot{\tilde{S}}(\rho)$ in Fig. 1 for two different temperatures, with each subsystem initially prepared in $|\uparrow\rangle$. To pinpoint the role played by the $\mathcal{S}_{A}-\mathcal{S}_{B}$ correlations in setting the nonmonotonic temporal trend of the heat flow [cf. Fig. 1(c)], we have studied $I(A: B)$. Figure 1(d) shows that the establishment of such correlations indeed acts as a precursor of the nonmonotonicity of the heat exchanged with the environment. In Ref. [22], we support these conclusions with the study of a multipartite system.

Indirect erasure.-We now analyze the dynamics of a bipartite system $\mathcal{S}$, in which the evolution of the two subsystems and their coupling to the subenvironments are treated on different footings: We include a mutual interaction between $\mathcal{S}_{A}$ and $\mathcal{S}_{B}$ while we assume that only $\mathcal{S}_{B}$ interacts with $\mathcal{R}$. This amounts to assuming that the information contained in $\mathcal{S}_{A}$ is first transferred to $\mathcal{S}_{B}$ and then damped into $\mathcal{R}$, a clear example of indirect information erasure. In practice we assume that between the $\mathcal{S}_{B}-\mathcal{R}_{n}$ and $\mathcal{S}_{B}-\mathcal{R}_{n+1}$ collisions, the two subsystems $\mathcal{S}_{A}$ and $\mathcal{S}_{B}$ interact. Equations (1) and (2) are still valid provided that $\hat{U} \rightarrow \hat{U}_{B} \hat{U}_{A B}$, where $\hat{U}_{B}$ (describing an $\mathcal{S}_{B}-\mathcal{R}_{n}$ collision) is the same as in the last section, while $\hat{U}_{A B}=e^{-i \hat{H}_{A B} \tau}$ regulates the joint evolution of the $\mathcal{S}_{A}-\mathcal{S}_{B}$ system. To go to continuous time, we expand each unitary operator to lowest order in $\tau$. Unlike the previous case, we

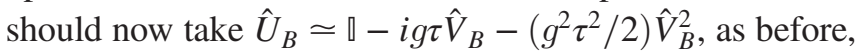
and $\hat{U}_{A B} \simeq \llbracket-i \tau \hat{H}_{A B}$. We thus find [22]

$$
\begin{aligned}
\dot{\rho}= & -i\left[J \hat{H}_{A B}, \rho\right] \\
& +\gamma \sum_{k j}\left\langle\hat{R}_{k} \hat{R}_{j}\right\rangle_{\eta^{\text {th }}}\left(\hat{S}_{B j} \rho \hat{S}_{B k}-\frac{1}{2}\left\{\hat{S}_{B k} \hat{S}_{B j}, \rho\right\}\right) .
\end{aligned}
$$

This is a Lindblad ME describing the Markovian dynamics of $\mathcal{S}$. The dynamics of $\mathcal{S}_{A}$, however, is in general nonMarkovian.
We now illustrate the indirect-erasure model with a specific example, where we set no constraint on the dimension of the Hilbert space of $\mathcal{S}_{B}$. In Ref. [22], we derive the form of Eq. (13) when $\mathcal{S}_{B}$ is a quantum harmonic oscillator resonantly coupled to a two-level subsystem $\mathcal{S}_{A}$ via the interaction Hamiltonian $\hat{H}_{A B}=\omega\left(\hat{q}^{2}+\hat{p}^{2}+\hat{\sigma}_{A z} / 2\right)+$ $J\left(\hat{q} \hat{\sigma}_{A x}+\hat{p} \hat{\sigma}_{A y}\right)$, with $\hat{q}$ and $\hat{p}$ the position and momentum quadrature operators of oscillator $\mathcal{S}_{B}$, respectively. An analogous model holds for the $\mathcal{S}_{B}-\mathcal{R}_{n}$ interaction, each subenvironment being a qubit. The bosonic nature of $\mathcal{S}_{B}$ allows us to explore the effects that multiple excitations in $\mathcal{S}_{B}$ have on both the heat flow and rate of entropy change. Indeed, as illustrated in Figs. 2(d) and 2(e), for an initially thermal harmonic oscillator at the same temperature of the bath, the differences with a finite-dimensional subsystem $\mathcal{S}_{B}$ are striking, and dependent on $\beta$. At low temperature, due to the excitation-conserving nature of the Hamiltonian, the dynamics of $\mathcal{S}$ is in fact identical to that of a two-qubit system, as only one excitation at most can be exchanged with $\mathcal{R}$. This is not the case when the temperature is raised, as excitations can be pumped into the harmonic oscillator at each collision [cf. Fig. 2(e)]. In Ref. [22] we study the behavior of the key quantities in our analysis against $J$ and $\gamma_{g}$. Interesting considerations on the nature of the dynamics of $\mathcal{S}_{A}$ are in order. Indeed, by tracing out the degrees of freedom of $\mathcal{S}_{B}$, we obtain a non-Markovian evolution due to the flowback of information into $\mathcal{S}_{A}$. Correspondingly, Eq. (7) is violated very quickly, as shown in Fig. 3, which again highlights the effects that the establishment of intrasystem correlations have on the efficiency of the information-to-energy conversion processes. (a)

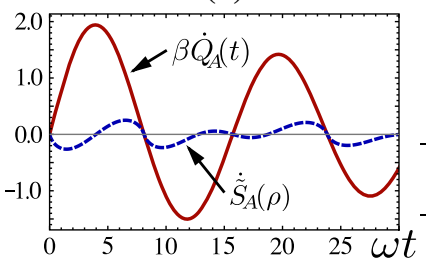

(b)

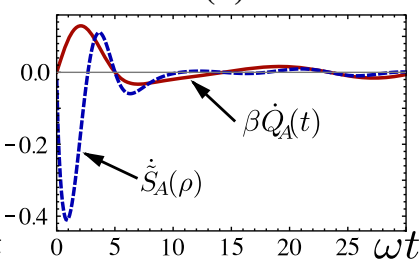

FIG. 3 (color online). We plot $\beta \dot{Q}_{A}(t)$ and $\dot{\tilde{S}}_{A}$ for the qubitoscillator configuration in the indirect-erasure case. In panel (a) $[(\mathrm{b})]$ we have used $\beta=10(\beta=0.5)$ and the parameters stated in Figs. 2(d) and 2(e). 
Similar conclusions hold when relaxing the key assumption of noninteracting subenvironments. The breakdown of such a condition would entail the establishment of memory effects within the environment and the occurrence of non-Markovianity in the $\mathcal{S}_{A}-\mathcal{S}_{B}$ dynamics. The violation of our formulation of Landauer's principle could thus be used to infer the nature of the bath itself.

Conclusions. - We have investigated the information-toenergy conversion processes in terms of collision-based models to describe the system-environment interaction, with the aim to go towards the microscopic formulation of Landauer's principle. In our approach we focused on the rate of entropy change and dissipated-heat flux involved in the overall erasure process. By doing so, we have been able to link the efficiency of the erasure process to the intrasystem correlations arising in the open dynamics of a multipartite system.

We thank T. J. G. Apollaro, A. Farace, O. Dahlsten, E. Lutz, and Y. Omar for discussions and suggestions. M. P. is grateful to J. Goold and K. Modi for fruitful discussions. We acknowledge financial support from the Department of Employment and Learning, the John Templeton Foundation (Grant No. 43467), MIUR-PRIN2010/11, and the EU Collaborative Project TherMiQ (Grant No. 618074). This work was partially supported by the COST Action MP1209.

[1] R. Landauer, IBM J. Res. Dev. 5, 183 (1961).

[2] A. Bérut, A. Arakelyan, A. Petrosyan, S. Ciliberto, R. Dillenschneider, and E. Lutz, Nature (London) 483, 187 (2012); Y. Jun, M. Gavrilov, and J. Bechhoefer, Phys. Rev. Lett. 113, 190601 (2014); A. O. Orlov, C. S. Lent, C. C. Thorpe, G. P. Boechler, and G. L. Snider, Jpn. J. Appl. Phys. 51, 06FE10 (2012).

[3] C. Bennett, Int. J. Theor. Phys. 17, 525 (1973); O. Penrose, Foundations of Statistical Mechanics: A Deductive Treatment (Pergamon, New York, 1970).

[4] M. Esposito, U. Harbola, and S. Mukamel, Rev. Mod. Phys. 81, 1665 (2009); M. Campisi, P. Hänggi, and P. Talkner, ibid. 83, 771 (2011).

[5] R. Dorner, S. R. Clark, L. Heaney, R. Fazio, J. Goold, and V. Vedral, Phys. Rev. Lett. 110, 230601 (2013); L. Mazzola, G. De Chiara, and M. Paternostro, ibid. 110, 230602 (2013); T. B. Batalhão, A. M. Souza, L. Mazzola, R. Auccaise, R. S. Sarthour, I. S. Oliveira, J. Goold, G. De Chiara, M. Paternostro, and R. M. Serra, ibid. 113, 140601 (2014); L. Mazzola, G. De Chiara, and M. Paternostro, Int. J. Quantum. Inform. 12, 1461007 (2014); F. Plastina, A. Alecce, T. J. G. Apollaro, G. Falcone, G. Francica, F. Galve, N. Lo Gullo, and R. Zambrini, Phys. Rev. Lett. 113, 260601 (2014); M. Campisi, R. Blattmann, S. Kohler, D. Zueco, and P. Hänggi, New J. Phys. 15, 105028 (2013); J. Goold, U. Poschinger, and K. Modi, Phys. Rev. E 90, 020101R (2014); A. J. Roncaglia, F. Cerisola, and J. P. Paz, Phys. Rev. Lett. 113, 250601 (2014); G. De Chiara, A. J. Roncaglia, and J. P. Paz, New J. Phys. 17, 035004 (2015).
[6] J. P. P. Silva, R. S. Sarthour, A. M. Souza, I. S. Oliveira, J. Goold, K. Modi, D. O. Soares-Pinto, and L. C. Céleri, arXiv:1412.6490.

[7] D. Kafri and S. Deffner, Phys. Rev. A 86, 044302 (2012); S. Deffner and C. Jarzynski, Phys. Rev. X 3, 041003 (2013); A. C. Barato and U. Seifert, Phys. Rev. Lett. 112, 090601 (2014); J. Parrondo, J. M. Horowitz, and T. Sagawa, Nat. Phys. 11, 131 (2015).

[8] J. Goold, M. Huber, A. Riera, L. del Rio, and P. Skrzypczyk, arXiv:1505.07835.

[9] D. Reeb and M. W. Wolf, New J. Phys. 16, 103011 (2014).

[10] J. Goold, M. Paternostro, and K. Modi, Phys. Rev. Lett. 114, 060602 (2015).

[11] S. Hilt, S. Shabbir, J. Anders, and E. Lutz, Phys. Rev. E 83, 030102 (2011).

[12] J. Rau, Phys. Rev. 129, 1880 (1963); R. Alicki and K. Lendi, Quantum Dynamical Semigroups and Applications, Lecture Notes in Physics Vol. 286 (Springer-Verlag, Berlin, 1987); M. Ziman, P. Štelmachovič, V. Bužek, M. Hillery, V. Scarani, and N. Gisin, Phys. Rev. A 65, 042105 (2002); V. Scarani, M Ziman, P. Štelmachovič, N. Gisin, and V. Bužek, Phys. Rev. Lett. 88, 097905 (2002); M. Ziman and V. Bužek, Phys. Rev. A 72, 022110 (2005); M. Ziman, P. Štelmachovič, and V. Bužek, Open Syst. Inf. Dyn. 12, 81 (2005); G. Benenti and G. M. Palma, Phys. Rev. A 75, 052110 (2007); G. Gennaro, G. Benenti, and G. M. Palma, Europhys. Lett. 82, 20006 (2008); G. Gennaro, G. Benenti, and G. M. Palma, Phys. Rev. A 79, 022105 (2009); G. Gennaro, S. Campbell, M. Paternostro, and G. M. Palma, ibid. 80, 062315 (2009).

[13] V. Giovannetti and G. M. Palma, Phys. Rev. Lett. 108, 040401 (2012); J. Phys. B 45, 154003 (2012).

[14] F. Ciccarello, G. M. Palma, and V. Giovannetti, Phys. Rev. A 87, 040103(R) (2013); F. Ciccarello and V. Giovannetti, Phys. Scr. T153, 014010 (2013).

[15] R. McCloskey and M. Paternostro, Phys. Rev. A 89, 052120 (2014).

[16] H.-P. Breuer and F. Petruccione, The Theory of Open Quantum Systems (Oxford University Press, Oxford, 2002).

[17] J. Spohn, J. Math. Phys. (N.Y.) 19, 1227 (1978); R. Alicki, J. Phys. A 12, L103 (1979); W. Pusz and S. L. Woronowicz, Commun. Math. Phys. 58, 273 (1978).

[18] S. Deffner and E. Lutz, Phys. Rev. Lett. 107, 140404 (2011); J. Anders and V. Giovannetti, New J. Phys. 15, 033022 (2013).

[19] The relation is easily proved considering the form of the equilibrium state $\rho^{\mathrm{eq}}$ and that, for a trace-preserving map, $\operatorname{Tr}[\dot{\rho}]=0$. Indeed, $\dot{S}\left(\rho \mid \rho^{\mathrm{eq}}\right)=\operatorname{Tr}\left[\dot{\rho} \ln \rho-\dot{\rho} \ln \rho^{\mathrm{eq}}\right]=\operatorname{Tr}[\dot{\rho} \ln \rho]+$ $\beta \operatorname{Tr}\left[\dot{\rho} \hat{H}_{\mathcal{S}}\right]+\ln \left(\operatorname{Tr}\left[e^{-\beta \hat{H}_{\mathcal{S}}}\right]\right) \operatorname{Tr}[\dot{\rho}]=-\dot{S}+\beta \operatorname{Tr}\left[\dot{\rho} \hat{H}_{\mathcal{S}}\right]=-\dot{S}+\beta \dot{E}$.

[20] V. Vedral, Rev. Mod. Phys. 74, 197 (2002).

[21] S. Lorenzo, A. Farace, F. Ciccarello, G. M. Palma, and V. Giovannetti, Phys. Rev. A 91, 022121 (2015).

[22] See Supplemental Material at http://link.aps.org/ supplemental/10.1103/PhysRevLett.115.120403, which includes Ref. [23].

[23] K. Stannigel, P. Rabl, and P. Zoller, New J. Phys. 14, 063014 (2012).

[24] M. A. Nielsen and I. L. Chuang, Quantum Computation and Quantum Information (Cambridge University Press, Cambridge, England, 2000).

[25] F. Herbut, J. Phys. A 37, 3535 (2004). 